\title{
A GEOMETRIC APPROACH TO THE REPRESENTATIONS OF THE FULL LINEAR GROUP OVER A GALOIS FIELD
}

\author{
BY \\ R. STEINBERG
}

1. Introduction. In this paper, methods are given for obtaining a large number of representations of $G \equiv G L(n, q)$, the group of all nondegenerate linear transformations of $S(n, q)$, the $n$-dimensional vector space over the Galois field $G F(q)$ of $q=p^{r}$ elements; this group will be considered equivalently as the group of all nondegenerate $n$ by $n$ matrices over the Galois field.

In $\$ 2$, through a favorable comparison of $G$ and $H$, the symmetric group on $n$ symbols, we obtain a basic set of $p(n)$ irreducible $\left({ }^{1}\right)$ characters of $G$ closely related to those of $H$. Among the characters obtained is one of degree $q^{n(n-1) / 2}$ which is of particular interest from the group theoretical and the modular representations $[1]\left({ }^{2}\right)$ points of view since $q^{n(n-1) / 2}$ is the highest power of a prime $p$ dividing the order $g$ of $G$. In $\$ 3$, the characters of this representation are computed explicitly.

In $\S 4$, by making use of linear characters of suitably chosen subgroups of $G$, a large number of irreducible characters of $G$ is obtained.

The methods used involve the elementary properties of finite group representations $\left({ }^{3}\right)$ and characters, especially of permutation representations, and the Frobenius formula for induced characters which enables one to find a character of a group if he knows one for a subgroup [2].

2 . We shall first determine $p(n)$ irreducible representations of $G$ by considering simple geometric properties of $S(n, q)$ or, briefly, of $S(n)$. Corresponding to a fixed partition of $n, n=\nu_{1}+\nu_{2}+\cdots+\nu_{n} \equiv(\nu)$, say, with $0 \leqq \nu_{1}$ $\leqq \nu_{2} \leqq \cdots \leqq \nu_{n}$, let $e(\nu)$ be a sequence of subspaces $S\left(\nu_{1}\right), S\left(\nu_{1}+\nu_{2}\right), \cdots$, $S\left(\nu_{1}+\nu_{2}+\cdots+\nu_{n-1}\right), S(n)$ which are such that each subspace is contained in the following one. The $e(\nu)$ 's are permuted by the elements of $G$, and hence we get a permutation representation $C(\nu)$ of $G$ of degree

$$
c(\nu)=\{n\} /\left\{\nu_{1}\right\}\left\{\nu_{2}\right\} \cdots\left\{\nu_{n}\right\},
$$

the number of $e(\nu)$ 's, where the notation used is

$$
[r]=q^{r-1}+q^{r-2}+\cdots+q+1, \quad\{r\}=\prod_{i=1}^{r}[i] .
$$

Presented to the Society, February 26, 1949; received by the editors December 18, 1948 and November 23, 1949 and, in revised form, February 3, 1951.

(1) $p(n)$ denotes the number of ways of dividing $n$ into non-negative integers.

(2) Numbers in brackets refer to the references at the end of the paper.

( ${ }^{8}$ An account of these properties and methods can be found in [3]. 
We shall find it convenient to define $[r]$ for all integers by

$$
[-r]=-q^{-r}[r] \text {. }
$$

Then, for any two integers $r$ and $s$, we have

$$
[r]-[s]=q^{s}[r-s] .
$$

Corresponding to the $p(n)$ partitions of $n$, we thus get $p(n)$ representations of $G$.

Now, let $\lambda_{i}=\nu_{i}+(i-1)$. Then, the $\lambda$ 's satisfy the relations

$$
\begin{gathered}
0 \leqq \lambda_{1}<\lambda_{2}<\cdots<\lambda_{n}, \\
\sum_{i=1}^{n} \lambda_{i}=n(n+1) / 2 .
\end{gathered}
$$

Also, let us define sgn $\left(\kappa_{1}, \kappa_{2}, \cdots, \kappa_{n}\right)$ to be 0 if two of the $\kappa^{\prime}$ s are equal and otherwise 1 or -1 according as $\kappa_{1}, \kappa_{2}, \cdots, \kappa_{n}$ form an even or an odd permutation of the $\kappa$ 's written in ascending order of magnitude.

Then our main result of this section is the following theorem.

Theorem 2.1. $\Gamma(\nu)=\sum_{\kappa} \operatorname{sgn}\left(\kappa_{1}, \kappa_{2}, \cdots, \kappa_{n}\right) C\left(\lambda_{1}-\kappa_{1}, \lambda_{2}-\kappa_{2}, \cdots, \lambda_{n}-\kappa_{n}\right)$ $=\sum_{\kappa} \operatorname{sgn}(\kappa) C(\lambda-\kappa)$ is an irreducible representation of $G$. (The summation is made as $\kappa_{1}, \kappa_{2}, \cdots, \kappa_{n}$ run through the $n$ ! permutations of the numbers $0,1,2, \cdots, n-1$; and $C(\lambda-\kappa)$ is defined to be 0 if any $\lambda_{i}-\kappa_{i}$ is negative.) Moreover, the $p(n)$ representations $\Gamma(\nu)$ so obtained are all distinct.

We shall first prove two lemmas of a geometric nature on which the proof of Theorem 2.1 depends.

Lemma 2.1. If $e^{(1)}$ and $e^{(2)}$ are two ${ }^{(4)} e\left(1^{n}\right)$ 's, there exist $n$ vectors $V_{1}, V_{2}, \cdots$, $V_{n}$ which, when taken in the order $V_{1} V_{2} \cdots V_{n}$, span $e^{(1)}$, and, taken in some other order $V_{p_{1}} V_{p_{2}} \cdots V_{p_{n}}$, span $e^{(2)}$.

We say that the ordered set of vectors $V_{1} V_{2} \cdots V_{n}$ spans $e^{(1)}=S^{(1)}(1)$, $S^{(1)}(2), \cdots, S^{(1)}(n)$ if $S^{(1)}(i)=\left\{V_{1}, V_{2}, \cdots, V_{i}\right\}$ for $i=1,2, \cdots, n$.

Proof of Lemma 2.1. The proof will be by induction, and, since the lemma is trivially true for $n=1$ or 2 , we shall assume $n \geqq 3$, and that the lemma is true for $n-1$.

Now $e^{(1)}$ and $e^{(2)}$ intersect $S^{(1)}(n-1)$ in two $e\left(1^{n-1}\right)$ 's which we shall denote by $e^{(1) '}$ and $e^{(2)^{\prime}}$. By the induction assumption, we can choose $n-1$ vectors $V_{1}, V_{2}, \cdots, V_{n-1}$ such that $V_{1} V_{2} \cdots V_{n-1}$ and $V_{p_{1}} V_{p_{2}} \cdots V_{p_{n-1}}$ span $e^{(1)^{\prime}}$ and $e^{(2)^{\prime}}$ respectively. If $S^{(1)}(n-1)$ contains $S^{(2)}(i)$ but not $S^{(2)}(i+1)$, choose $V_{n}$ to be any vector in $S^{(2)}(i+1)$ but not in $S^{(2)}(i)$. Then $V_{1} V_{2} \cdots V_{n}$ and $V_{p_{1}} V_{p_{2}} \cdots V_{p_{i}} V_{n} V_{p_{i+1}} \cdots V_{p_{n-1}}$ span $e^{(1)}$ and $e^{(2)}$ respectively, and the lemma is proved.

$\left({ }^{4}\right)\left(1^{n}\right)$ denotes the partition of $n$ into $n$ ones. 
We next note an analogy between $G$ and $H$, the symmetric group on $n$ symbols, and make use of this analogy. Corresponding to the general partition $(\nu)$ of $n$, we define an $s(\nu)$ to be an entity consisting of the symbols 1 to $n$ in any order, the first $\nu_{1}$ symbols being bracketed together, the next $\nu_{2}$ being bracketed together, and so forth. Two $s(\nu)$ 's are considered to be the same if one can be obtained from the other by permuting the symbols in their separate brackets; for example, a typical $s\left(2^{2}\right)$ is $12,34 \equiv 21,34 \equiv 12,43 \equiv 21,43$. The $s(\nu)$ 's are permuted by the elements of $H$, and hence furnish a permutation representation $D(\nu)$ of $H$ of degree

$$
d(\nu)=n ! / \nu_{1} ! \nu_{2} ! \cdots \nu_{n} !,
$$

the number of $s(\nu)$ 's (cf. (2.1)).

It should be noted here that $D(\nu)$ is the representation of $H$ induced by the unit representation of the subgroup $H(\nu)=H \nu_{1} \times H \nu_{2} \times \cdots \times H \nu_{n}$, where $H_{i}$ is a symmetric group on $i$ symbols, and that $C(\nu)$ is the representation of $G$ induced by the unit representation of the subgroup $G(\nu)$ consisting of matrices with square blocks of degrees $\nu_{1}, \nu_{2}, \cdots, \nu_{n}$ (in this order) down the main diagonal and zeros above them.

Lemma 2.2. The number of classes of $(e(\nu), e(\mu))$ 's is equal to the number of classes of $(s(\nu), s(\mu))$ 's.

Here, we mean that $\left(e^{(1)}(\nu), e^{(1)}(\mu)\right)$ and $\left(e^{(2)}(\nu), e^{(2)}(\mu)\right)$, for example, belong to the same class if there is an element of $G$ taking $e^{(1)}(\nu)$ into $e^{(2)}(\nu)$ and $e^{(1)}(\mu)$ into $e^{(2)}(\mu)$.

Proof of Lemma 2.2. For any $(e(\nu), e(\mu))$, we supply the missing subspaces, if necessary, and then by Lemma 2.1 we choose $n$ vectors $V_{1}, V_{2}, \cdots$, $V_{n}$ such that $V_{1} V_{2} \cdots V_{n}$ and $V_{p_{1}} V_{p_{2}} \cdots V_{p_{n}}$ span $e(\nu)$ and $e(\mu)$ respectively. We then associate the class containing $(e(\nu), e(\mu))$ with the class containing the $(s(\nu), s(\mu))$ given by

$\left(12 \cdots \nu_{1}, \nu_{1}+1 \cdots \nu_{1}+\nu_{2}, \cdots, n\right.$

$$
\left.p_{1} p_{2} \cdots p_{\mu_{1}}, p_{\mu_{1}+1} \cdots p_{\mu_{1}+\mu_{2}}, \cdots p_{n}\right) .
$$

Since there is a nondegenerate linear transformation taking any $n$ vectors which span $S(n)$ into any other $n$ vectors which span $S(n)$, we see that two $(e(\nu), e(\mu))$ 's belong to the same class if and only if the corresponding $(s(\nu)$, $s(\mu))$ 's do, and this proves the lemma.

Geometrically, we have the following result.

COROLlARY. There is a nondegenerate linear transformation taking any $(e(\nu), e(\mu))$ into any other $(e(\nu), e(\mu))$ with the same degrees of intersection of all corresponding pairs of subspaces.

Now, let $\psi(\nu), \phi(\nu)$, and $\chi(\nu)$ be the characters of $C(\nu), D(\nu)$, and $\Gamma(\nu)$ 
respectively. Then, the permutation representations of the $(e(\nu), e(\mu))$ 's and the $(s(\nu), s(\mu))$ 's are given by the Kronecker products $C(\nu) \times C(\mu)$ and $D(\nu)$ $X D(\mu)$, and thus their characters by $\psi(\nu) \psi(\mu)$ and $\phi(\nu) \phi(\mu)$ respectively. But, for any permutation representation of character $\chi$ of a group of order $g$, $g^{-1} \sum \chi(x)$ is the number of times $\chi$ contains the unit character, that is, the number of classes of transitivity. (The summation is made over all elements $x$ of $G$.) This remark together with Lemma 2.2 proves the following lemma.

Lemma(5) 2.3. $g^{-1} \sum_{x \in G} \psi(\nu, x) \psi(\mu, x)=h^{-1} \sum_{y \in H} \phi(\nu, y) \phi(\mu, y)$.

Proof of Theorem 2.1. Let $\lambda_{i}=\nu_{i}+i-1$ and $\sigma_{i}=\mu_{i}+i-1$, and let $\kappa_{1}, \kappa_{2}, \cdots, \kappa_{n}$ and $\rho_{1}, \rho_{2}, \cdots, \rho_{n}$ be permutations of the numbers $0,1,2, \cdots$, $n-1$.

Then (6),

$$
\begin{aligned}
g^{-1} \sum_{x \in G} \chi(\nu, x) \chi(\mu, x) & =g^{-1} \sum_{x \in G} \sum_{\kappa} \operatorname{sgn}(\kappa) \psi(\lambda-\kappa, x) \sum_{\rho} \operatorname{sgn}(\rho) \psi^{*}(\sigma-\rho, x) \\
& =h^{-1} \sum_{\nu \in H} \sum_{\kappa} \operatorname{sgn}(\kappa) \phi(\lambda-\kappa, y) \sum_{\rho} \operatorname{sgn}(\rho) \phi^{*}(\sigma-\rho, y),
\end{aligned}
$$

by the definitions and Lemma 2.3 respectively.

But, $\sum_{\kappa} \operatorname{sgn}(\kappa) \phi(\lambda-\kappa)$ is the character of $\Delta(\nu)=\sum_{\kappa} \operatorname{sgn}(\kappa) D(\lambda-\kappa)$, and it was proved by Frobenius [3] that the $p(n)$ representations $\Delta(\nu)$ of $H$ are irreducible and distinct. Thus

$$
g^{-1} \sum_{x \in Q} \chi(\nu, x) \chi^{*}(\mu, x)=\delta_{(\nu)(\mu)}
$$

Thus, if in addition $\chi(\nu, E)>0$, where $E$ is the identity element of $G, \chi(\nu)$, being an integral linear combination of characters, will be an irreducible character.

Now,

$$
\begin{aligned}
\chi(\nu, E) & =\sum_{\kappa} \operatorname{sgn}(\kappa)\{n\} /\left\{\lambda_{1}-\kappa_{1}\right\}\left\{\lambda_{2}-\kappa_{2}\right\} \cdots\left\{\lambda_{n}-\kappa_{n}\right\} \\
& =\{n\} \operatorname{det}\left|\left\{\lambda_{i}-(j-1)\right\}^{-1}\right| \quad\left(\text { where }\{r\}^{-1} \equiv 0 \text { if } r<0\right) \\
& =\frac{\{n\} \operatorname{det}\left|\left[\lambda_{i}\right]\left[\lambda_{i}-1\right] \cdots\left[\lambda_{i}-(j-2)\right]\right|}{\left\{\lambda_{1}\right\}\left\{\lambda_{2}\right\} \cdots\left\{\lambda_{n}\right\}} .
\end{aligned}
$$

To evaluate this, let us consider the determinant

$$
\left|X_{i}\left(\frac{X_{i}-[1]}{q}\right)\left(\frac{X_{i}-[2]}{q^{2}}\right) \cdots\left(\frac{X_{i}-[j-2]}{q^{j-2}}\right)\right| .
$$

It is of degree $n(n-1) / 2$ in the $n$ indeterminates $X_{1}, X_{2}, \cdots, X_{n}$ and van-

(5) $\psi(\nu, x)$ denotes the character in $\psi(\nu)$ of the element $x$ of $G$.

$(8) *$ denotes conjugate complex. 
ishes if $X_{i}=X_{j}, i \neq j$. Hence, it is a constant( $\left(^{7}\right)$ times $\Delta\left(X_{1}, X_{2}, \cdots, X_{n}\right)$ $=\prod_{i<j}\left(X_{j}-X_{i}\right)$, and a perusal of the coefficient of $X_{2} X_{3}^{2} \cdots X_{n}^{n-1}$ shows this constant to be $q^{-n(n-1)(n-2) / 3}$. Since this determinant reduces to the one in (2.8) if $X_{i}=\left[\lambda_{i}\right]$, we finally get

$$
\begin{aligned}
\gamma(\nu) & =\chi(\nu, E) \\
& =q^{-n(n-1)(n-2) / 3}\{n\} \Delta\left(\left[\lambda_{1}\right],\left[\lambda_{2}\right], \cdots,\left[\lambda_{n}\right]\right) /\left\{\lambda_{1}\right\}\left\{\lambda_{2}\right\} \cdots\left\{\lambda_{n}\right\} .
\end{aligned}
$$

This is positive since $\lambda_{i}<\lambda_{j}$ for $i<j$. This completes the proof of Theorem 2.1, and the equation (2.9) furnishes us with the degree $\gamma(\nu)$ of $\Gamma(\nu)$.

As an immediate consequence of Theorem 2.1 and Lemma 2.3, we get the following corollaries.

Corollary 1. $C(\nu)$ and $D(\nu)$ split into irreducible representations in exactly the same manner; that is, if $C(\nu)=\sum_{(\mu)} k(\nu, \mu) \Gamma(\mu)$, then $D(\nu)$ $=\sum_{(\mu)} k(\nu, \mu) \Delta(\mu)$.

Corollary 2. Let $\left(\nu_{1}\right),\left(\nu_{2}\right), \cdots,\left(\nu_{n}\right)$ be further partitions of $\nu_{1}, \nu_{2}, \cdots, \nu_{n}$, let $C$ be the representation of $G$ induced by the representation $\Gamma\left(\nu_{1}\right) \times \Gamma\left(\nu_{2}\right)$ $\times \cdots \times \Gamma\left(\nu_{n}\right)$ of $G(\nu)$, and let $D$ be the corresponding representation of $H$. Then $C$ and $D$ split into irreducible representations in exactly the same manner.

We can give an alternate representation to that given by (2.5) for the partitions of $n$, and this will lead to a simpler formula for the degree $\gamma(\nu)$ of $\Gamma(\nu)$. Among the numbers $\lambda_{1}, \lambda_{2}, \cdots, \lambda_{n}$ there are a certain number, say $r$, which are not less than $n$. Denote these by $n+b_{1}, n+b_{2}, \cdots, n+b_{r}$. Denote the remaining $\lambda$ 's by $n-1-a_{r+1}, n-1-a_{r+2}, \cdots, n-1-a_{n}$. Let $n-1-a_{1}, n-1-a_{2}, \cdots, n-1-a_{r}$ be the rest of the numbers $0,1, \cdots$, $n-1$ so that the $a$ 's are a permutation of the numbers $0,1, \cdots, n-1$. Then, we can represent the partition by the two sets of integers $a_{1}, a_{2}, \cdots, a_{r}$ and $b_{1}, b_{2}, \cdots, b_{r}$. These $a$ 's and $b$ 's can be ordered so that

$$
0 \leqq a_{1}<a_{2}<\cdots<a_{r}, \quad 0 \leqq b_{1}<b_{2}<\cdots<b_{r} .
$$

Because of (2.5), we have also

$$
\sum_{i=1}^{r} a_{i}+\sum_{i=1}^{r} b_{i}=n-r .
$$

Conversely, it can be shown that two sets of $r$ integers satisfying the relations (2.10) and (2.11) correspond to a unique partition of $n$. The partition obtained from a given one by interchanging the $a$ 's and $b$ 's we shall call the conjugate partition $\left({ }^{8}\right)$.

(7) Hereafter we shall call this the Vandermonde determinant.

${ }^{8}$ ) One can represent a partition diagrammatically by the lattice points $(i, j)$ which satisfy $1 \leqq j \leqq \nu_{n-i}$ and alternately define the conjugate partition to be the one represented by the points $(j, i)$. These two definitions are equivalent. 
Then, by a computation similar to that used by Frobenius [3], and making use of (2.4), we can show that

$$
\begin{aligned}
& \gamma(\nu)=q^{r(r-1) / 2+\sum_{i=1}^{r}\left(a_{i}+1\right) a_{i} / 2} \\
& \frac{\left.\{n\} \Delta\left(\left[a_{1}\right],\left[a_{2}\right], \cdots, \mid a_{r}\right]\right) \Delta\left(\left[b_{1}\right],\left[b_{2}\right], \cdots,\left[b_{r}\right]\right)}{\left\{a_{1}\right\}\left\{a_{2}\right\} \cdots\left\{a_{r}\right\}\left\{b_{1}\right\}\left\{b_{2}\right\} \cdots\left\{b_{r}\right\} \prod_{\alpha, \beta=1}^{r}\left[a_{\alpha}+b_{\beta}+1\right]}
\end{aligned}
$$

From this a direct computation shows that the degress $\gamma(\nu), \gamma\left(\nu^{\prime}\right)$ of representations corresponding to conjugate partitions $(\nu),\left(\nu^{\prime}\right)$ are polynomials in $q$ related by the following formula:

$$
\gamma\left(\nu^{\prime}, q\right)=q^{n(n-1) / 2} \gamma\left(\nu, q^{-1}\right) .
$$

As an important special case, if $(\nu)=\left(0^{n-1} n\right)$ and $\left(\nu^{\prime}\right)=\left(1^{n}\right)$, then $\gamma(\nu)=1$ (since $\Gamma(\nu)$ in this case is the unit representation) and $\gamma\left(\nu^{\prime}\right)=q^{n(n-1) / 2}$. As previously stated, this last representation is of group-theoretical importance and in the next section we shall compute its characters explicitly.

In closing this section, a remark on the analogy between $G$ and $H$ seems to be in order. Instead of considering $G$ as a group of linear transformations of a vector space, we could consider $G$ as a collineation group of a finite $(n-1)$-dimensional geometry. If $q=1$, the vector space fails to exist but the finite geometry does exist and, in fact, reduces to the $n$ vertices of a simplex with a collineation group isomorphic to $H$. Hence, if we put $q=1,[r]=r$, and $\{r\}=r$ ! in (2.1), (2.9), (2.12), and (2.13), we get corresponding results for $H$.

3. In this section, the characters of the representation of degree $q^{n(n-1) / 2}$ of $G$ mentioned in the previous section will be found. These characters are given by Theorem 3.1 at the end of this section.

The determination will depend on a number of lemmas which we proceed to state and prove.

By the generating function of an element $x$ of $G$, we shall mean the symmetric polynomial

$$
f(q, t)=\sum f(\nu) t^{(\nu)},
$$

where the sum is taken over all partitions $(\nu)$ of $n, f(\nu)$ is the number of $e(\nu)$ 's left fixed by $x$, and $t^{(\nu)}=t_{1}^{\nu_{1}} t_{2}^{\nu_{2}} \cdots t_{n}^{\nu_{n}}$.

Lemma 3.1. Let $A$ be an irreducible matrix of degree $m$ and En the unit matrix of degree $n$ (over $G F(q)$ ). Then, if the generating function of $E n$ is $f(q, t)$, that of $E n \times A$ is $f\left(q^{m}, t^{m}\right)$.

Proof. Let the field $G F(q)$ be extended to $G F\left(q^{m}\right)$. In this field $A$ can be transformed to a diagonal matrix $R$ whose main-diagonal element $\stackrel{\dot{s}}{\text { are }}$ 
conjugate relative to $G F(q)$. Then, after a further simple transformation, we get $E n \times A$ similar to $R \times E n$. The latter matrix leaves fixed all of the vectors of $m$ disjoint conjugate (relative to $G F(q)) S(n)$ 's and all subspaces spanned by such vectors. If we take $m$ conjugate vectors, one from each $S(n)$, we get a fixed real $S(m)$. Similarly, the only fixed real $S(\mathrm{rm})$ 's are spanned by conjugate $S(r)$ 's. Since each $S(n)$ is a $G F\left(q^{m}\right)$ vector space, the lemma is proved.

Lemma 3.2. Let $f_{1}, f_{2}, f$ be the generating functions for $A_{1}, A_{2}, A_{1} \dot{+} A_{2}$, where $A_{1}$ and $A_{2}$ are matrices which, when written in canonical form, have no common constituents. Then $f=f_{1} f_{2}$.

This lemma follows from the definitions and from the fact that any fixed $S(i)$ of $A_{1}+A_{2}$ is obtained as the join of a fixed $S(j)$ in the space of $A_{1}$ and a fixed $S(i-j)$ in the space of $A_{2}$, this representation being unique.

LeMma 3.3. Let $f_{1}, f_{2}$ be homogeneous polynomials of degree $m, n$ respectively, symmetric in the $m+n$ variables $t_{i}$, and let $\Delta_{1}, \Delta_{2}, \Delta$ be the Vandermonde determinants in $t_{1}, t_{2}, \cdots, t_{m} ; t_{m+1}, t_{m+2}, \cdots, t_{m+n} ; t_{1}, t_{2}, \cdots, t_{m+n}$ respectively. Let $c_{1}, c_{2}, c$ be the coefficients of $t_{1} t_{2}^{2} \cdots t_{m}^{m}, t_{m+1} t_{m+2}^{2} \cdots t_{m+n}^{n}, t_{1} t_{2}^{2} \cdots t_{m+n}^{m+n}$ in $f_{1} \Delta_{1}, f_{2} \Delta_{2}, f \Delta$ respectively. Then $c_{1} c_{2}=c$.

Proof. If we consider $f_{2} \Delta$, it is evident that any term with a repeated index will vanish, and, since we are interested only in those terms in which each $t_{i}$ occurs to a power of at most $m+n$, we need consider only terms of the sort $t_{2} t_{3}^{2} \cdots t_{m}^{m-1} t_{m+1}^{m+1} \cdots t_{m+n}^{m+n}$. The coefficient of this term is $c_{2}$. If we multiply by $f_{1}$, we readily see that the terms involving $t_{m+1}^{m+1} \cdots t_{m+n}^{m+n}$ are $c_{2} f_{1} \Delta_{1} t_{m+1}^{m+1} \cdots$ $t_{m+n}^{m+n}$. Hence the lemma is established.

Lemma 3.4. Let $f(t)$ be a homogeneous polynomial of degree $n$, symmetric in the $n$ variables $t_{1}, \cdots, t_{n}$, and let $\Delta_{1}, \Delta$ be the Vandermonde determinants in $t_{1}, t_{2}, \cdots, t_{n}$ and $t_{1}, t_{2}, \cdots, t_{m n}$ respectively. Then the coefficient of $t_{1} t_{2}^{2} \cdots t_{n}^{n}$ in $f(t) \Delta_{1}$ is equal to $(-1)^{(m-1) n}$ times the coefficient of $t_{1} t_{2}^{2} \cdots t_{m n}^{m n}$ in $f\left(t^{m}\right) \Delta$.

This lemma is evident if one considers how the $t_{1} t_{2}^{2} \cdots t_{m n}^{m n}$ term is obtained when $f\left(t^{m}\right)$ is multiplied by $\Delta$.

Lemma 3.5. For the matrix En, the coefficient of $t_{1} t_{2}^{2} \cdots t_{n}^{n}$ in $f \Delta$ is $q^{n(n-1) / 2}$.

This follows from $\S 2$ and the following statement.

This coefficient, in the case of $E n$, gives the degree of the representation under consideration, and, in general, the character of the element in this representation.

Lemma 3.6. For a matrix which cannot be reduced to diagonal form in any extension of $G F(q)$, the coefficient of $t_{1} t_{2} \cdots t_{n}$ in $f \Delta$ is 0 .

This follows from a theorem due to R. Brauer and C. Nesbitt [1] to the 
effect that the character of any $p$-singular element $\left({ }^{9}\right)$ is 0 in an irreducible representation of order divisible by $p^{r}$, where $p^{r}$ is the highest power of a prime $p$ which divides the order of the group.

From these lemmas and the statement in Lemma 3.5, we get the following theorem.

TheOREM 3.1. For the element $E n \times A$, where $A$ is irreducible and of degree $m$, the character is $(-1)^{(m-1) n} q^{m n(n-1) / 2}$. For any element $x$ of $G$ which is made up of blocks of the type $E n \times A$, the character is obtained by multiplying the $(-1)^{(m-1) n} q^{m n(n-1) / 2}$ 's for the various blocks. For any p-singular element, the character is 0 .

4. We can make use of the $p(n)$ basic irreducible representations $\Gamma(\nu)$ to determine a large set of irreducible representations of $G$.

We first note that there are $q-1$ linear representations of $G$ corresponding to the powers of the determinants of the elements $x$ of $G$. Thus if $\rho$ is a primitive $(q-1)$ th root of unity in $G F(q)$ and $\epsilon$ is one in the field of complex numbers, and if $\operatorname{det} x=\rho^{\alpha}$, these representations are given by $x \rightarrow \epsilon^{\alpha u}, u$ $=1,2, \cdots, q-1$.

If we multiply $\Gamma(\nu)$ by each of these representations, we get $q-1$ irreducible representations which we shall denote by $\Gamma_{u}(\nu)$.

Let us now return to our general partition $n=\nu_{1}+\nu_{2}+\cdots+\nu_{n}$ and partition each $\nu_{i}$ further: $\nu_{i}=\sum \nu_{i j}$. We shall denote these partitions by $\left(\nu_{i}\right)$. Let the partition of $\nu_{i}$ have $k_{i 1}$ ones, $k_{i 2}$ twos, and so forth, and let $k=\sum k_{i j}$. Corresponding to these partitions, we can take the Kronecker product of representations of the type $\Gamma_{u}(\nu)$ of the constituents of the subgroup $G(\nu)$ of $G$ and get a representation of $G$ which we can write as $\times \prod_{i=1}^{n} \Gamma_{u_{i}}\left(\nu_{i}\right)$. This representation of $G(\nu)$ induces one in $G$ which we shall call $\Gamma$.

ThEOREM 4.1. If no two u's are equal, $\Gamma$, is irreducible. Moreover, the number of representations so obtained (by varying the $u$ 's and keeping the $\nu$ 's fixed) is $(q-1)(q-2) \cdots(q-k) / \prod k_{i j} !$. The degree of each is

$$
\gamma=\frac{\gamma\left(\nu_{1}\right) \gamma\left(\nu_{2}\right) \cdots \gamma\left(\nu_{n}\right)\{n\} .}{\left\{\nu_{1}\right\}\left\{\nu_{2}\right\} \cdots\left\{\nu_{n}\right\}}
$$

Due to the complexities of notation, the proof will not be given here. It depends on a computation similar to that used by Frobenius [3] in his determination of the characters of the symmetric group.

A combinatorial argument shows that the number of different degrees of characters obtained by this method has as generating function $h(t)$ $=\prod_{i=1}^{\infty}\left(1-t^{i}\right)^{-p(i)}$. I conjecture that the total number of different degrees has as generating function $\prod_{i=1}^{\infty} h\left(t^{i}\right)$. For $n=1,2,3,4$, this method gives

(9) A $p$-singular element is one of order divisible by $p$. 
characters of $1,3,6,14$ distinct degrees, and the total $\left({ }^{10}\right)$ number of distinct degrees is $1,4,8,22$ respectively.

\section{REFERENCES}

1. R. Brauer and C. Nesbitt, On the modular characters of groups. Ann. of Math. vol. 42 (1941) pp. 556-590.

2. G. Frobenius, Über Relationen zwischen den Charakteren einer Gruppe und denen ihrer Untergruppen, Preuss. Akad. Wiss. Sitzungsber. (1898) pp. 501-515.

3. - Über die Charaktere der symmetrische Gruppe, Preuss. Akad. Wiss. Sitzungsber. (1900) pp. 516-534.

4. F. D. Murnaghan, The theory of group representations, Baltimore, 1938.

5. R. Steinberg, Representations of the linear fractional groups, Thesis, 1948, University of Toronto Library.

UNIVERSITY OF CALIFORNIA, Los Angeles, Calif.

(10) All of the characters of $G$ for $n=1,2,3,4$ have been determined [5]. 